\title{
Vorticity Intensification and Transition to Turbulence in the Three-Dimensional Euler Equations
}

\author{
John B. Bell and Daniel L. Marcus \\ Lawrence Livermore National Laboratory, Livermore, CA 94551, USA
}

Received November 18, 1991

\begin{abstract}
The evolution of a perturbed vortex tube is studied by means of a second-order projection method for the incompressible Euler equations. We observe, to the limits of grid resolution, a nonintegrable blowup in vorticity. The onset of the intensification is accompanied by a decay in the mean kinetic energy. Locally, the intensification is characterized by tightly curved regions of alternating-sign vorticity in a $2 n$-pole structure. After the first $L^{\infty}$ peak, the enstrophy and entropy continue to increase, and we observe reconnection events, continued decay of the mean kinetic energy, and the emergence of a Kolmogorov $\left(k^{-5 / 3}\right)$ range in the energy spectrum.
\end{abstract}

\section{Introduction}

The regularity of solutions to the three-dimensional Euler equations continues to be an area of active research. That very steep gradients exist on very small length scales is beyond dispute; that the vorticity increases wildly as the volume of its support collapses onto a fractal set can be persuasively demonstrated [8]; that, for smooth initial data, solutions to the three-dimensional Euler equations become singular in finite time is an issue that remains open to debate, speculation, and controversy $[6,17,3,22,24,25]$.

The importance of such an event is obvious; it would dominate the vorticity dynamics and play a critical role in the turbulent energy cascade, thus providing a heuristic link between the geometry of incipient singularities and the dynamics of the intertial range. Therefore, the manner in which vorticity and strain couple to induce strong local vorticity intensification, the time scale on which it occurs, and the vorticity dynamics in the post-intensification regime are all issues of fundamental interest.

Chorin [8] observed that an inviscid vorticity distribution represented as a collection of vortex tubes becomes singular in finite time, and that the Hausdorff dimension of the support of the vorticity collapses to a value of roughly 2.5 . Siggia 
[26] performed further vortex filament calculations, with the conclusion that counter-sign vortices will merge in finite time, becoming antiparallel at their point of closest approach. This has inspired several numerical studies of colliding vortex tubes $[22,27]$ and fueled speculation as to the role of vortex reconnection in the turbulent energy cascade $[1,17,25]$.

The picture of the vorticity distribution that has emerged is of a collection of stretched, folded, and pinched vortex tubes [9]. This has been supported by experimental observation of tightly curved (hairpin) vortices in turbulent boundary layers [15] and free shear layers [20]. Moin et al. [19] have simulated the emergence of a hairpin vortex from a perturbed vortex layer and have shown that a curved vortex filament can evolve into a vortex ring in the presence of viscosity and mean shear, thus linking experimental observations of hairpin vortices with observations of vortex rings in the outer regions of a turbulent boundary layer. Hairpin vortices have been associated with turbulent bursts [18] and there has been speculation as to the connection between hairpin vortices and the formation of singularities [10,23]. Recently, Chorin and Akao [11] have proved (in a statistical mechanics context) that hairpin structures must occur, and are generic to turbulent flow.

In this paper, we study the evolution of a perturbed vortex tube in a triply periodic domain by means of a new finite difference method for the threedimensional Euler equations. The flow is visualized by following the evolution of a tracer function initialized on the surface of the tube. As the flow evolves, we calculate the $L^{\infty}$ norm of the vorticity, the enstrophy, the entropy of the vorticity magnitude, the mean kinetic energy, the energy spectrum, and the moments of the diagonal components of the deformation tensor. The choice of diagnostics is motivated by a desire to explore the following questions: What role do strong vorticity intensification evens have in the transfer of energy to the small scales? What is the local structure of such an event and how is that manifest in the global (statistical) properties of the flow?

We show that as $t$ approaches a critical time $t^{*},\|\omega\|_{\infty}$ increases as $1 /\left(t-t^{*}\right)$, until the finite grid can no longer support the intensification. Accompanying the onset of the intensification is a decay in the mean kinetic energy and the emergence of approximately a decade of Kolmogorov $\left(k^{-5 / 3}\right)$ range in the energy spectrum. The velocity statistics are highly non-Gaussian. The intensification is localized to tightly curved regions whose tips have a complex structure of alternating-sign vortex elements. The enstrophy and entropy continue to increase beyond the first $\|\omega\|_{\infty}$ peak, suggesting that vortex-stretching continues to dominate the dynamics. The results imply a breakdown of the discrete solution to the Euler equations and a viscous regularization, roughly analogous to that of shocks in Burgers' equation.

\section{Governing Equations, Initial Data, and Numerics}

We solve the three-dimensional Euler equations for incompressible flow:

$$
\begin{gathered}
U_{t}+U \cdot \nabla U=-\nabla P, \\
\nabla \cdot U=0,
\end{gathered}
$$

where $U=(u, v, w), P$ is the pressure, and $-1 / 2 \leqq x \leqq 1 / 2,-1 / 2 \leqq y \leqq 1 / 2,-1 / 2 \leqq z$ $\leqq 1 / 2$. The initial data represents a hollow-cored vortex tube, or cylindrical shear 
layer, subject to a small transverse perturbation in the form of a wide, flat Gaussian bump. Thus,

$$
U_{0}=\left(\tanh \left[\frac{\left(\varrho-\sqrt{y^{2}+z^{2}}\right)}{\delta}\right], 0, \varepsilon e^{-\beta\left(x^{2}+y^{2}\right)}\right) .
$$

The values of the numerical parameters $\varrho, \delta, \varepsilon$, and $\beta$ are, for the calculations presented herein, $0.15,0.0333,0.05$, and 15.0 . The tracer function, $\phi$, satisfies an advection equation, $\phi_{t}+U \cdot \nabla \phi=0$, and is initialized with a Gaussian profile that peaks at the inflection point of the hyperbolic tangent in the $u_{0}$ field, i.e. $\phi_{0}=e^{-\kappa\left(\varrho-\sqrt{\left.y^{2}+z^{2}\right)}\right.}$, where $\kappa=500$, and $\varrho$ is the same as in Eq. (1).

The equations are solved by means of a second-order projection method $[4,5]$, a higher-order extension of Chorin's projection algorithm [7]. Advectiondiffusion equations are solved without enforcing the incompressibility constraint, and the resulting velocity field is projected onto a divergence-free subspace. We incorporate a second-order, upstream-centered differencing procedure, based on ideas first introduced by Colella [14] for numerical solutions to systems of hyperbolic conservation laws. Respecting the characteristic structure of nonlinear advection provides a robust treatment of nonsmooth data without introducing spurious oscillations, even in the limit of vanishing viscosity. Monotonicity is preserved by means of a slope-limiting procedure that introduces a nonlinear dissipation, providing a local and selective treatment for the subgrid scales. These features make the method especially well-suited for flows with large spatiotemporal inhomogeneities and a wide range of length scales. Further details regarding the numerics can be found in $[4,5]$.

\section{Results}

\subsection{Flow Visualization}

Figure $1 \mathrm{a}-1$ shows volume renderings of the tracer field described in the previous section. The tracer was rendered with an opacity profile scaled to the tracer magnitude, so that the frames show the evolution of the material surface of the original vortex tube. The calculations were carried out on a uniform $128^{3}$ grid.

The first frame is at $t=0.8$; up until this time, the growth of the perturbation has been slow and unspectacular, manifest in the formation of patches of straininduced secondary vorticity on the top and bottom of the tube, offset, respectively, to the right and left of the origin (as governed by the direction of the mean flow). These structures intensify as the tube further deforms, acquiring definition, interweaving, and wrapping themselves around the primary vortex in a helical manner. The secondary vortices continue to intensify and begin to flatten into ribbon-like structures, themselves exhibiting a tendency towards helical deformation. By this time, the primary vortex has taken the shape of an inverted " $U$ " at either end, with thin ribbon-like parallel walls thickening to tubular structures at the base and tips, rising in the middle to form a topologically complicated knot of tightly curved vorticity (Fig. 1f).

It is worthwhile at this point to pause a moment and note some of the largescale features of the flow: a predominance of hairpin structures and a tendency in some regions towards flattening and helical deformation of vortex tubes, while in other regions, the vortices appear to retain a substantial core structure. The 

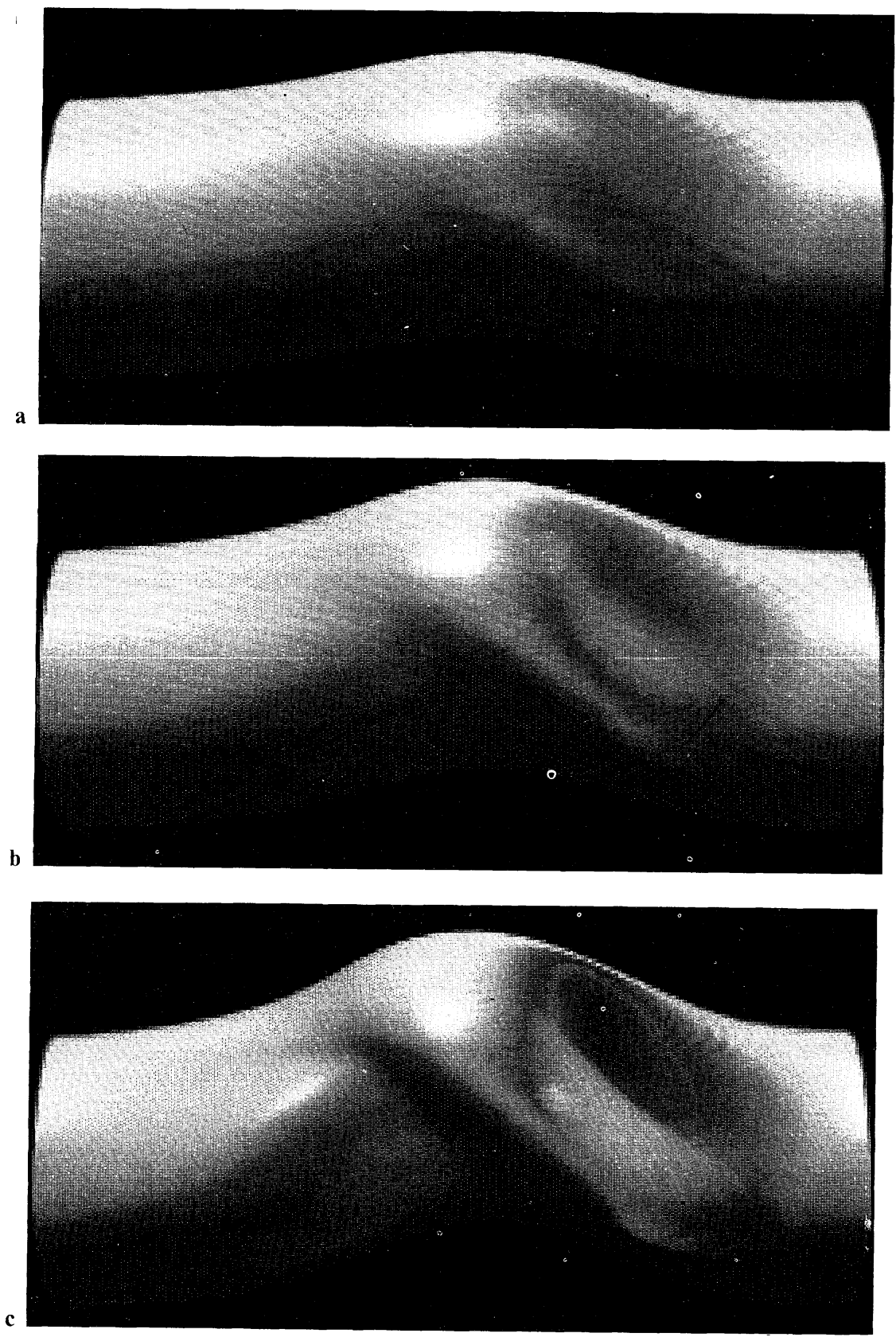

Fig. 1a-l. Evolution of tracer field; $t=0.8-(0.1)-1.9$ 

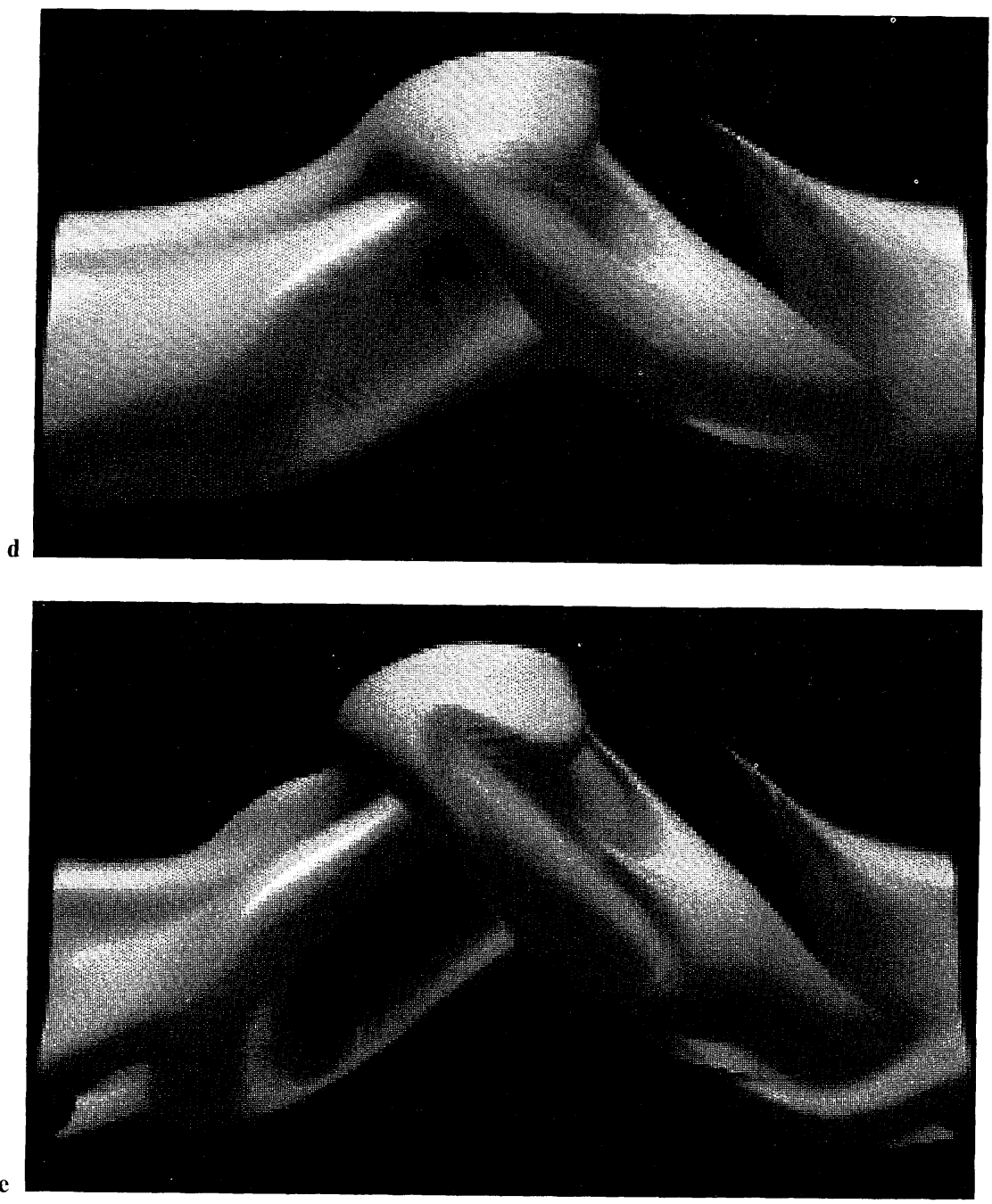

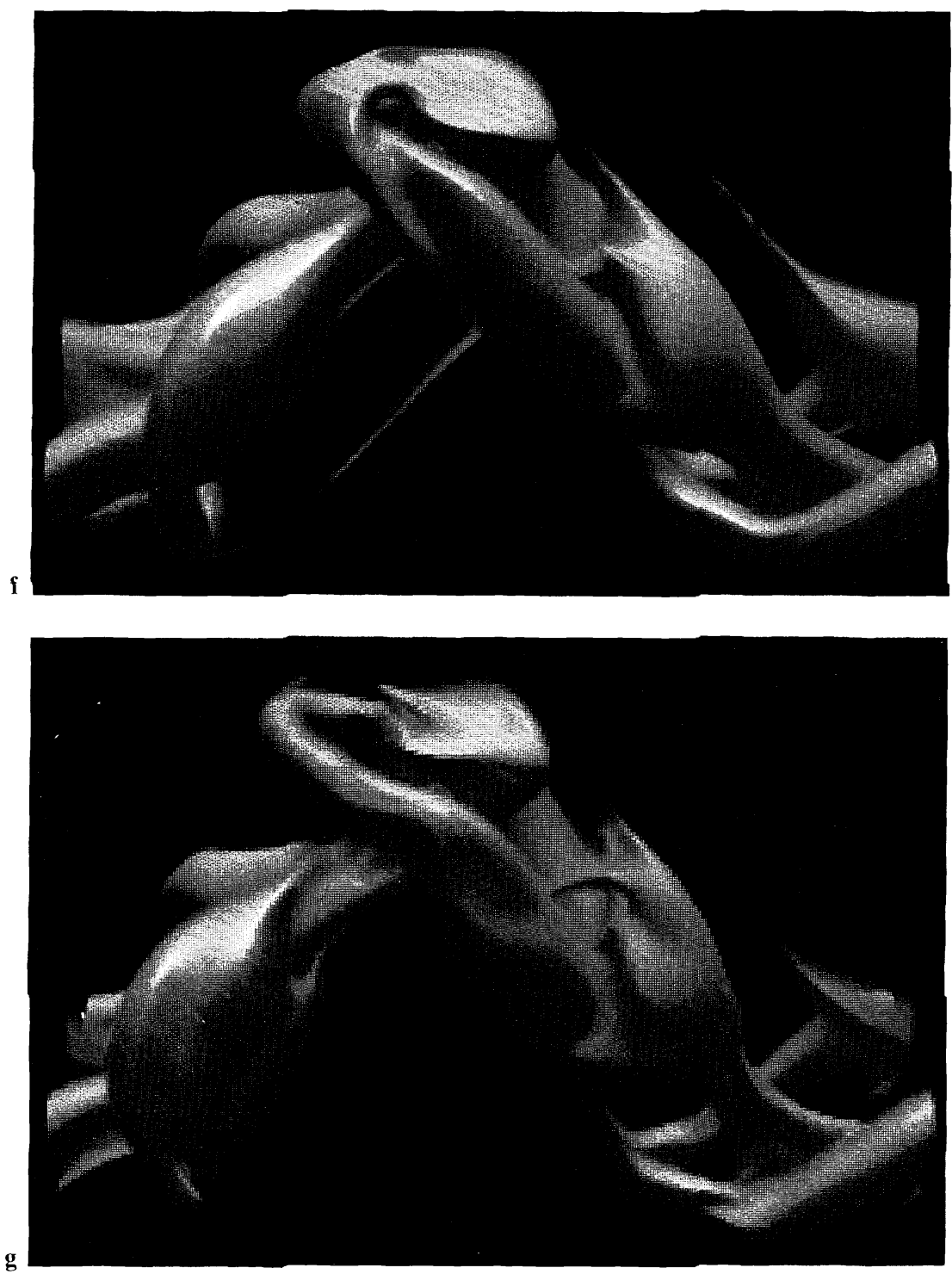

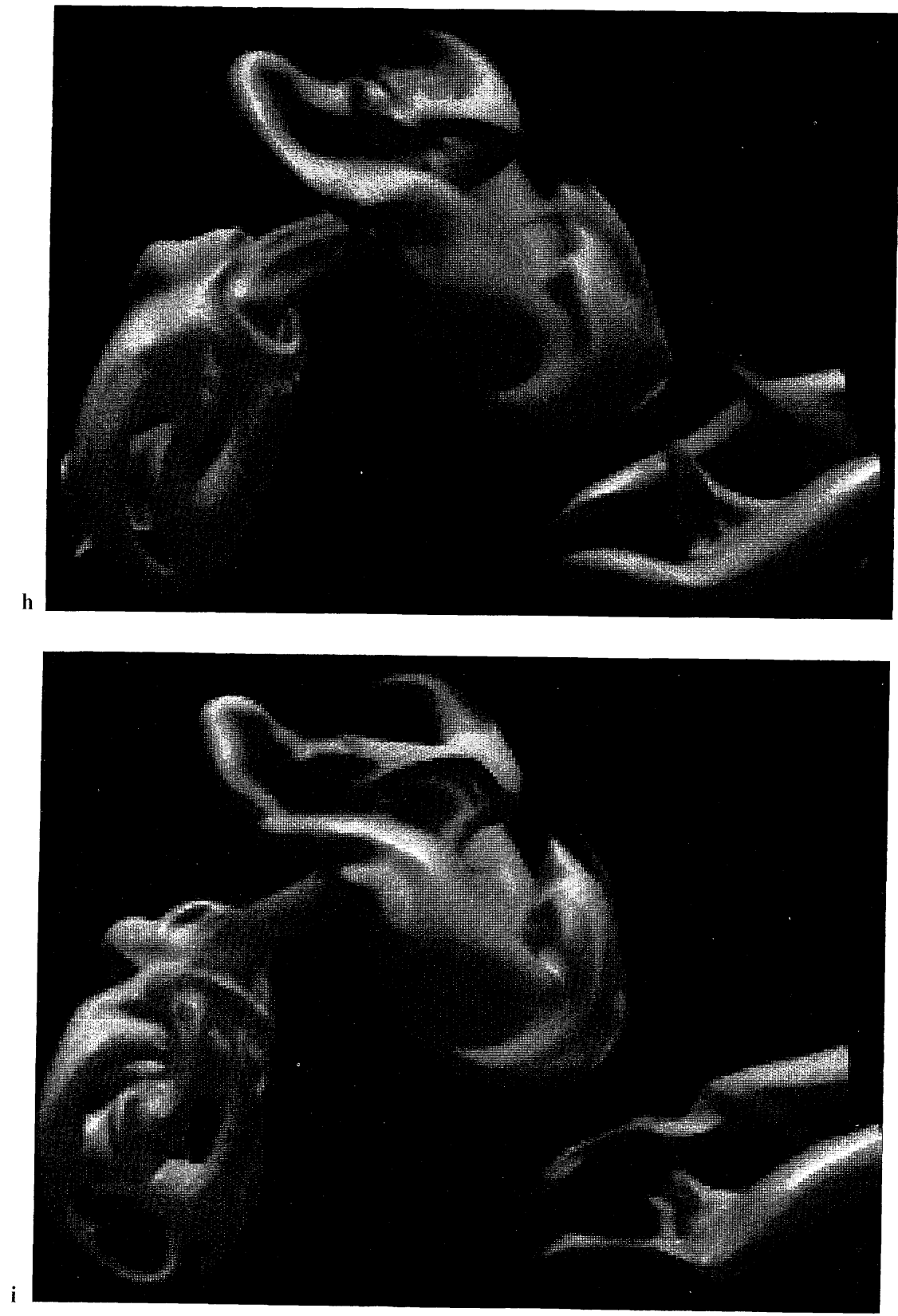

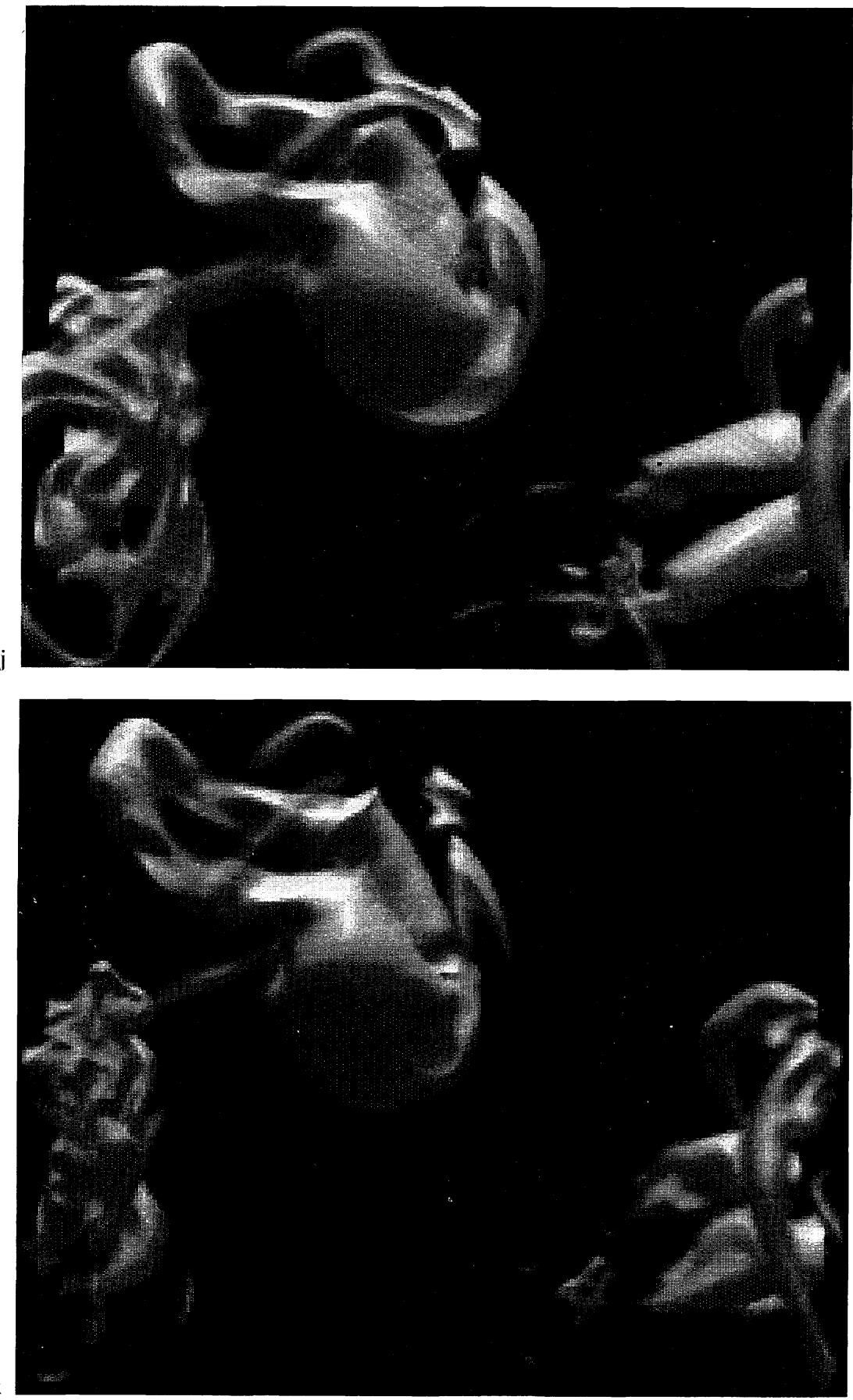


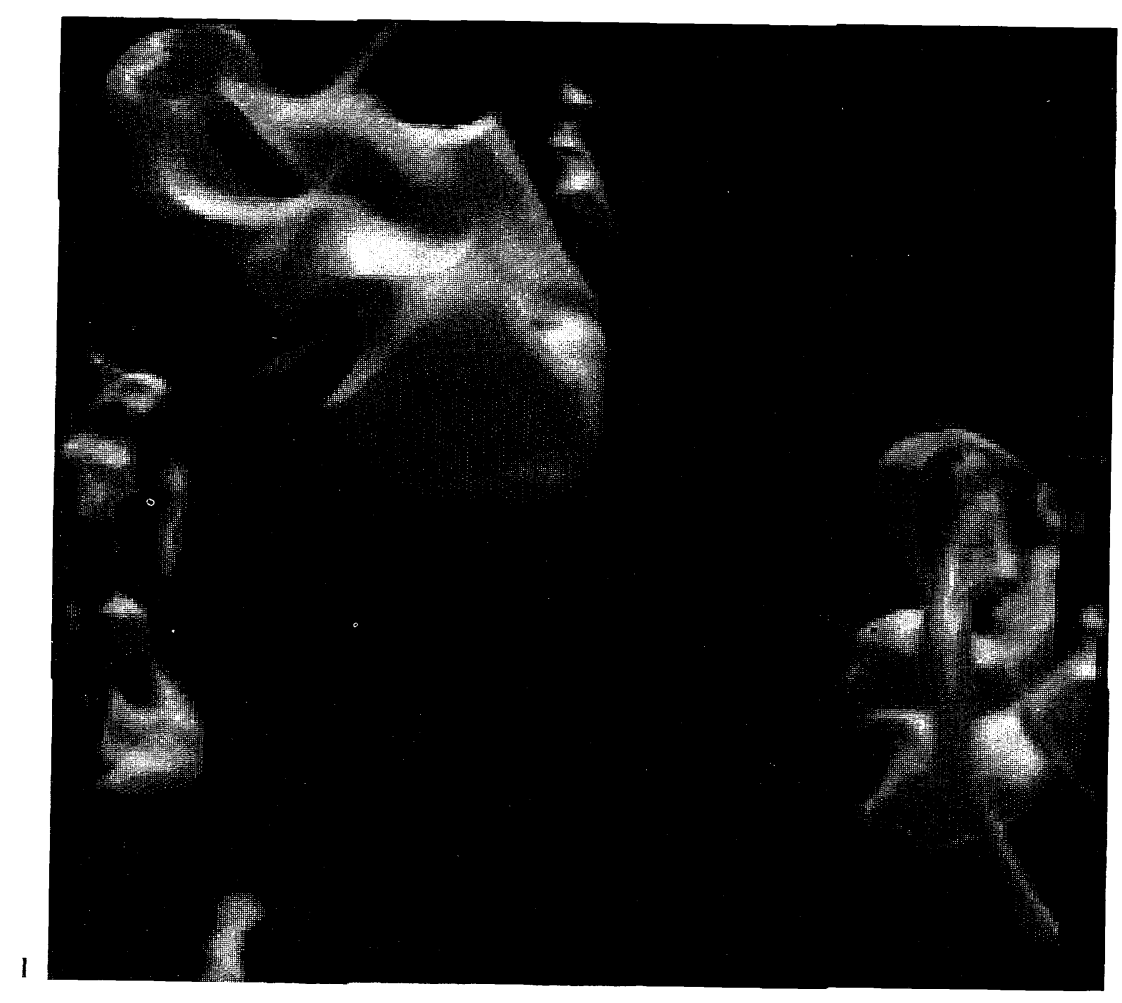

tendency towards "sheetification" of vorticity has been observed in other numerical simulations [22] and has been associated with the saturation of vorticity intensification in viscous flow simulations of vortex reconnection [25]. We will show later that the intensification we observe has a different local structure.

Figure $1 \mathrm{~h}$ shows the flow field shortly after the onset of the vorticity intensification. There are tightly curved structures at large and intermediate scales connected by highly attenuated regions. In the final frames, we observe what appear to be multiple reconnection events. Eventually, the flow takes on the appearance of a series of rough, staggered rings.

These volume renderings of the support of the vorticity are useful in that they promote a heuristic understanding of how a material surface in the flow evolves. Unfortunately, such a representation suppresses the sign-sense of the vorticity, which is essential to an understanding of the dynamics. In Fig. 2, we show contour plots of the vorticity components on orthogonal planes intersecting at the origin. It should be recalled, however, that this flow is highly three-dimensional, and these frames only represent "snapshots" of structures passing through the plane.

The left column shows the $z$-component of the vorticity in the $x-y$ plane. It is here that we can most clearly see the characteristic Kelvin-Helmholtz rollup of the vortex lines, particularly in the early stages. It is complicated by the entrainment of 
countersign vorticity and soon breaks up into two discrete large-scale structures which themselves exhibit a richness of small-scale detail, showing both thin, stretched sheets and dense-cored tubes of alternating-sign vorticity.

In the middle column, the $y$-component of the vorticity in the $x-z$ plane is shown. The smooth vortex lines associated with the primary shear flow are stretched and sheared, giving birth to the small intense vortices that characterize the flow at later times. The integrity of the initial vorticity distribution is maintained until the tube deforms enough so that vorticity from the lower surface is entrained by the counter-sign vorticity above, inducing the formation of energetic small scales.

The column on the right shows the component of vorticity parallel to the initial axis of the jet. As the perturbation grows, symmetry about the horizontal plane is lost, and patches of countersign vorticity are pushed toward the top of the jet, forming the knot-like structure seen in the tracer field. Intense local velocity fields arise due to the close proximity of countersign vortex patches, which induce further stretching, and so on.

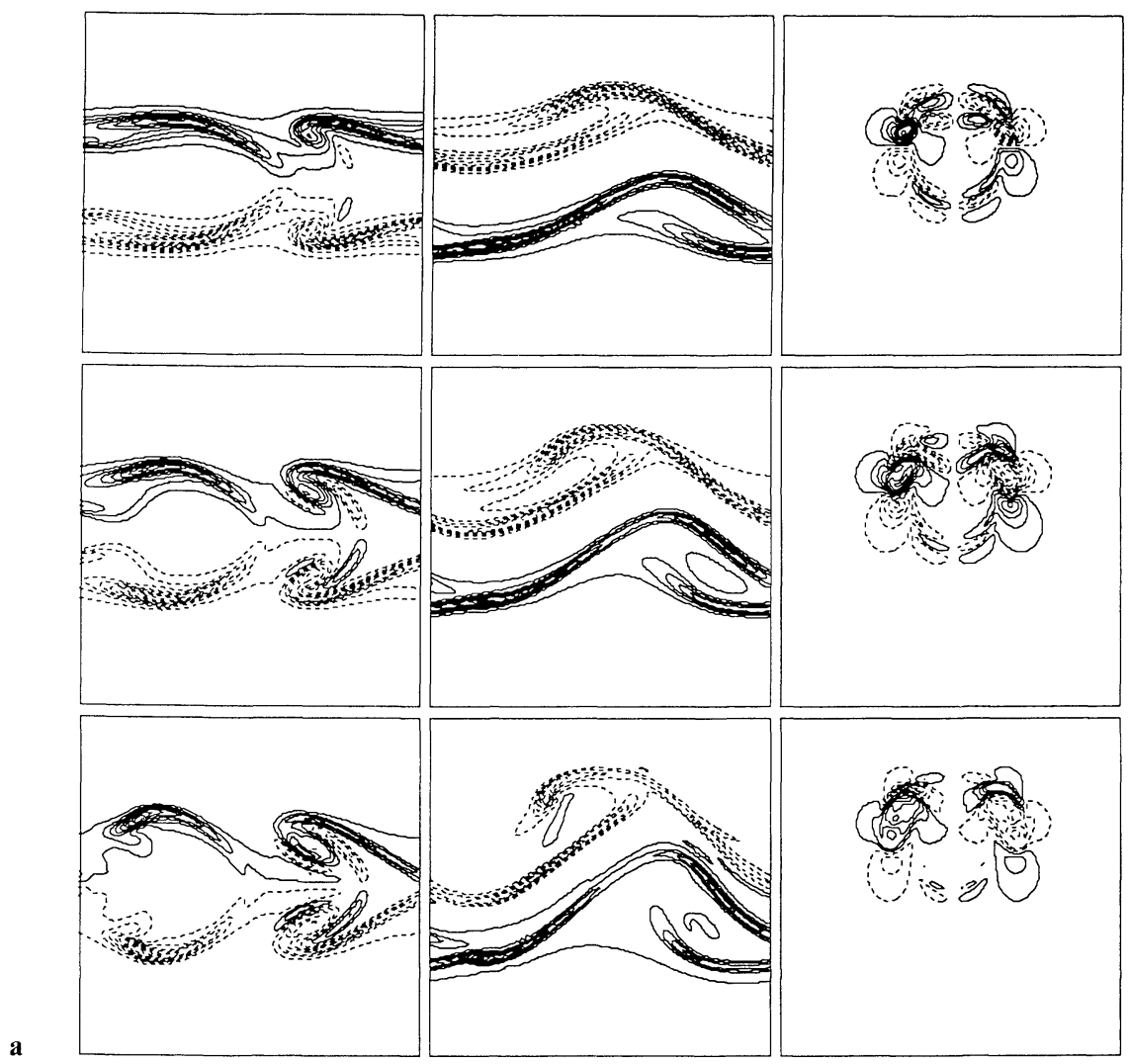

Fig. 2a, b. Contours of vorticity components on orthogonal planes through the origin; $t=1.0(0.1)-1.5$. Left column, $\omega_{z}$; center column, $\omega_{y}$; right column, $\omega_{x}$ 
At later times, the flow becomes so complicated that simple mechanistic descriptions of the evolving dynamics are of limited utility. However, some general observations can be made. Figure 3 shows isosurface plots of the vorticity magnitude for $|\omega|=0.5$ at $t=2.0,2.4$, and 2.8 , respectively. The vorticity is clearly not space-filling. We observe a broadening range of length scales with much smallscale structure, an increasing tendency towards isotropy, and a continued predominance of hairpin structures.

\section{2. $\|\omega\|_{\infty}$, Enstrophy, and the Local Structure of the Intensification}

Figure 4 shows the time evolution of $\|\omega\|_{\infty}$ for $32^{3}, 64^{3}$, and $128^{3}$ grids. Its value roughly doubles as the grid refinement is doubled. This indicates that, to the limit of grid resolution, we are capturing the asymptotic range of a finite-time "blowup." It is unknown if this intensification will saturate out at the higher wavenumbers resolvable on a finer grid, or if in the limit of infinite resolution $\|\omega\|_{\infty}$ would become unbounded. Singular or not, this vorticity intensification is an event of some significance, and the signature of a complex local structure. In
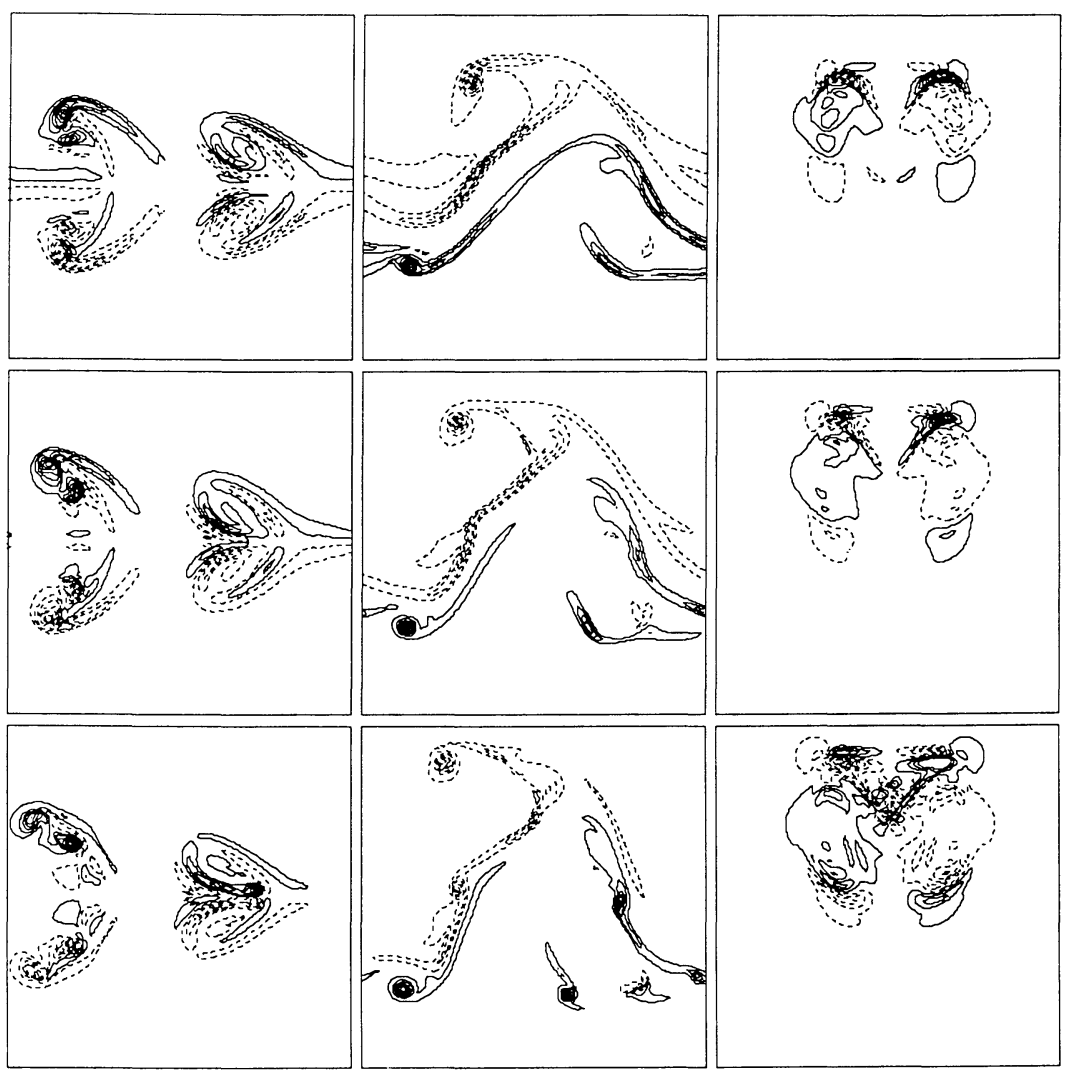


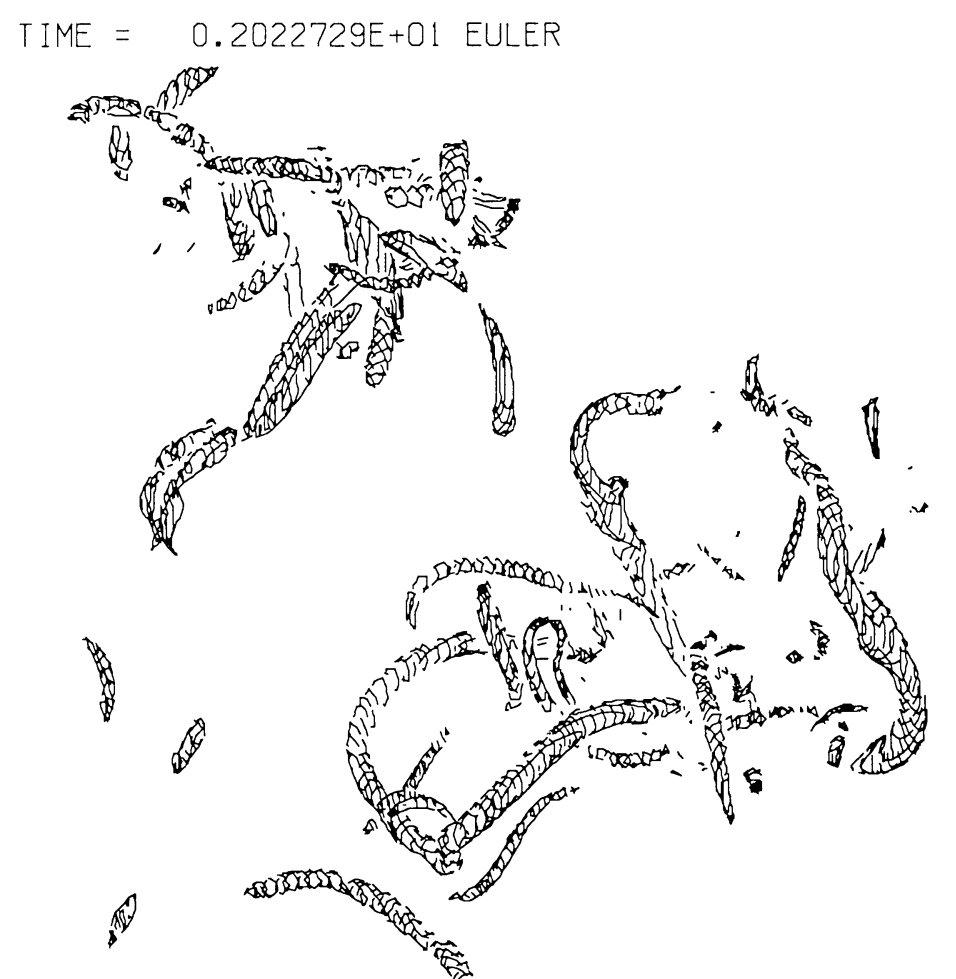

a

舟
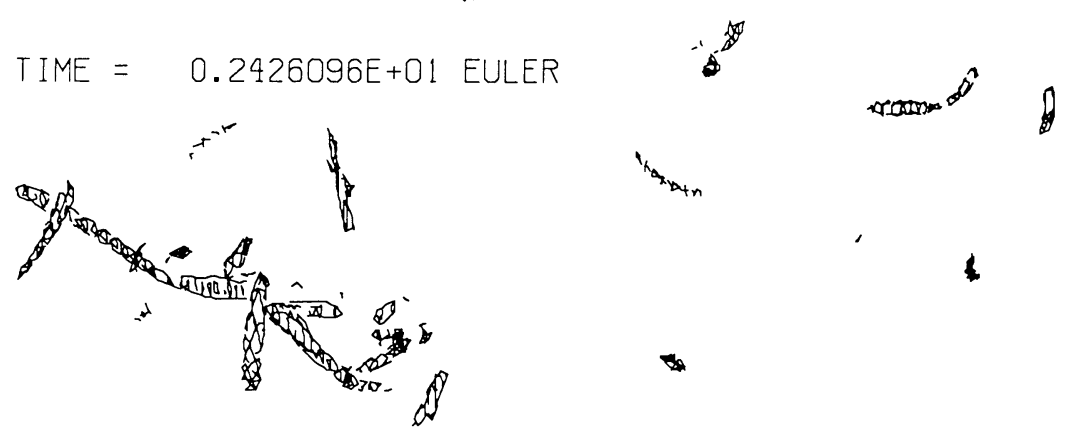

$\operatorname{sos}$

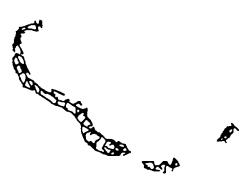

b

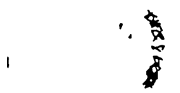




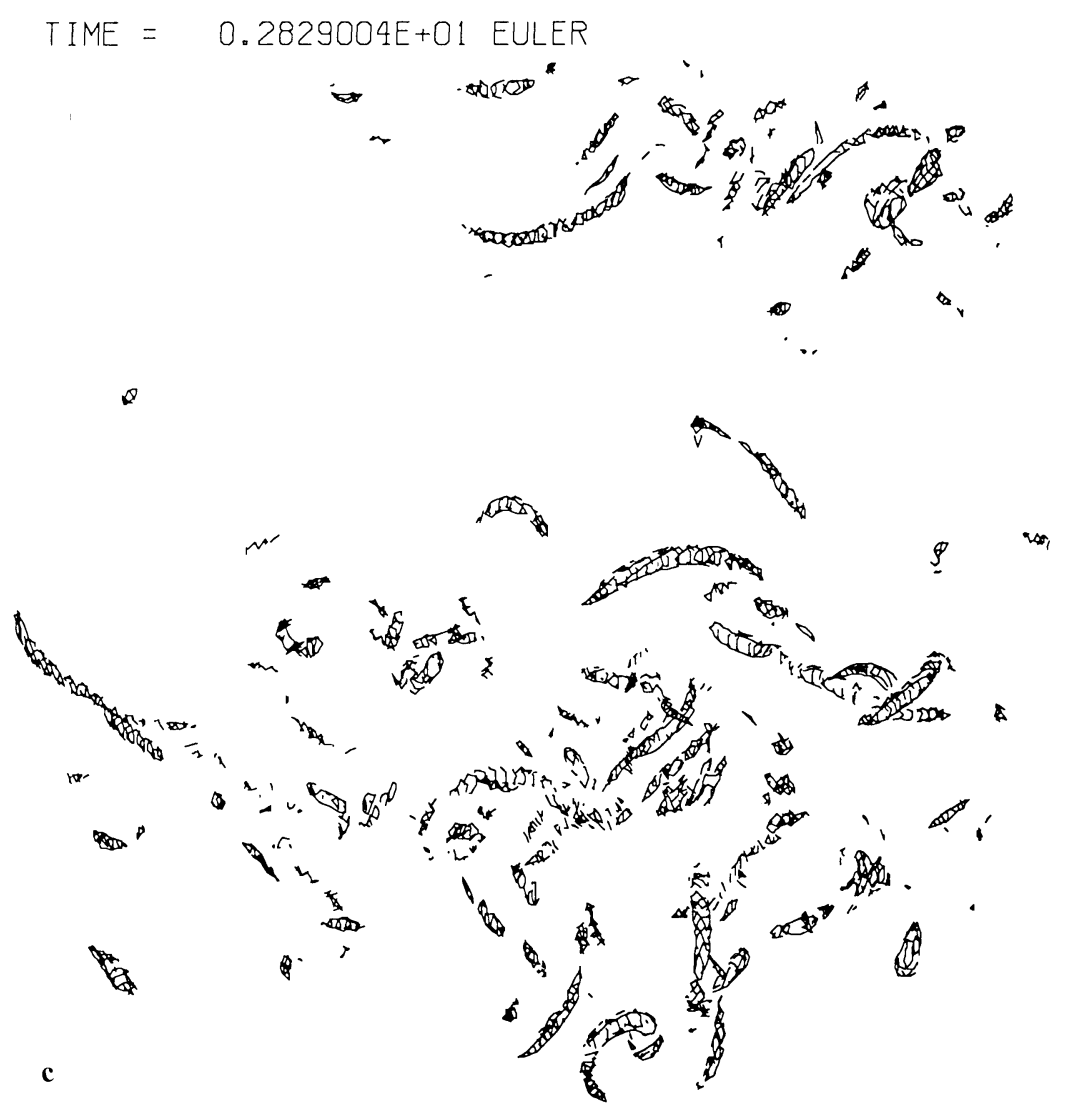

Fig. 3a-c. a $|\omega|=0.5$ isosurface; $t=2.0$. b $|\omega|=0.5$ isosurface; $t=2.4$. c $|\omega|=0.5$ isosurface; $t=2.8$

Fig. 5, we show the evolution of the enstrophy, defined as $Z=\int_{\Omega} \omega^{2} d \Omega$. We observe an increase that, in its early stages, follows the growth of $\|\omega\|_{\infty}$. The continued growth of the enstrophy after the first $L^{\infty}$ peak indicates that vortex stretching continues to dominate the dynamics [12].

At the highest resolution we observe a second $L^{\infty}$ peak at roughly $t=2.5$. This suggests a possible scenario for transition to turbulence as a cascade of vorticity intensification events, and lends credence to speculation regarding the connection between singularities, turbulent bursts, and intermittency.

The work of Beale, Kato, and Majda [3] provides a theoretical context for this discussion. They prove that if a finite time singularity occurs for the threedimensional Euler equations, it occurs in the $L^{\infty}$ norm and that the blow-up is nonintegrable. We have compared our results with a nonintegrable test function, $C\left(t^{*}-t\right)^{-1}$, where $t^{*}=1.568$ (Fig. 6). It is seen that, locally,

$$
\|\omega\|_{\infty} \sim \frac{1}{t-t^{*}} .
$$

Figure 7 shows a volume rendering of the magnitude of the vorticity field near the first $L^{\infty}$ peak $(t=1.5)$. The vorticity intensification is highly localized, concentrating on hairpin structures. As before, in order to get a true sense of the 


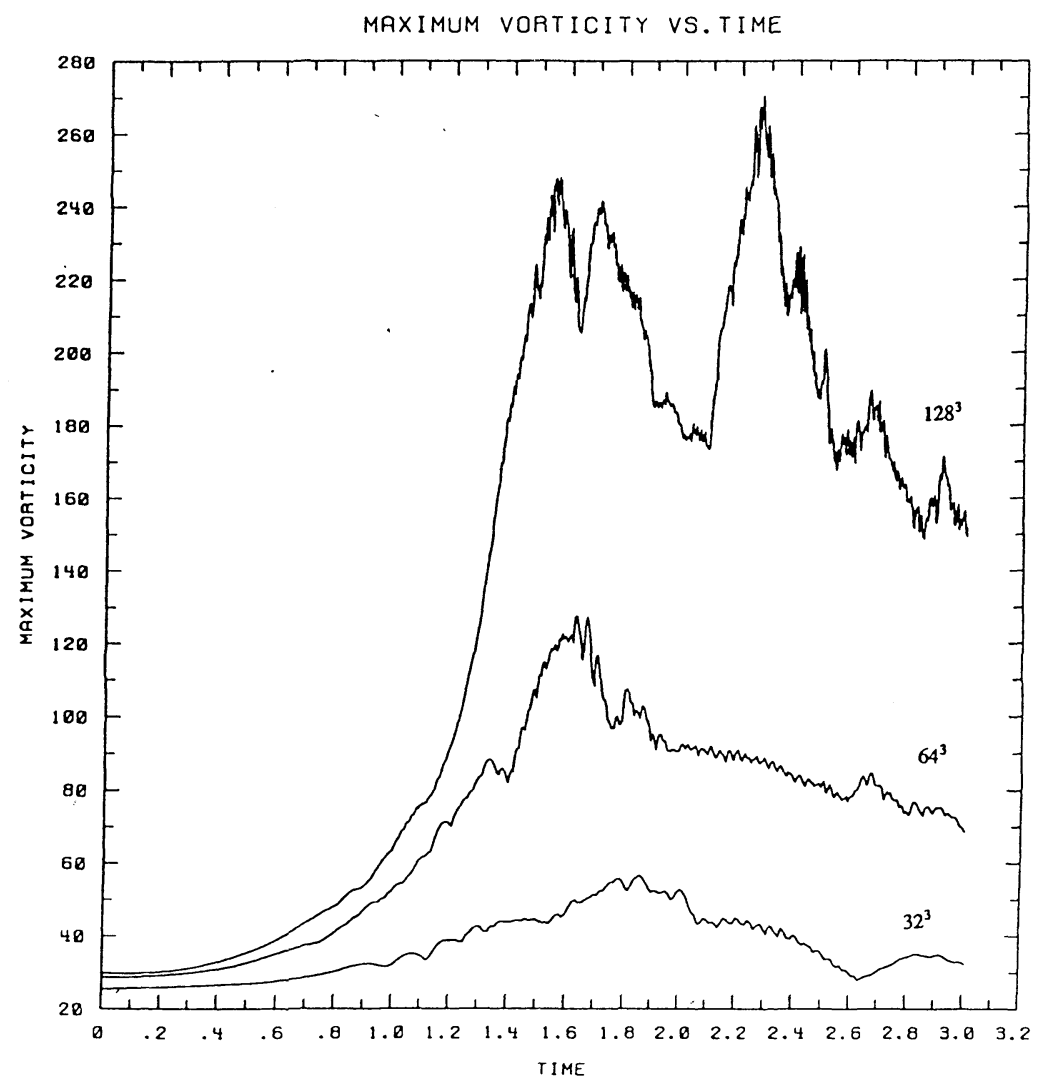

Fig. 4. $\|\omega\|_{\infty}$ vs. time; $32^{3}, 64^{3}$, and $128^{3}$ grids

vorticity dynamics, we plot contours of vorticity components on two-dimensional slices through the region of maximum intensification near the time of the first $L^{\infty}$ peak $(t=1.5)$. Figure 8 shows $\omega_{z}\left(x, y ; Z_{\omega_{\max }}\right)$; in Fig. 9 we plot $\omega_{x}\left(y, z ; X_{\omega_{\max }}\right)$. The tip of the hairpin seen in Fig. 7 is in fact a complicated $2 n$-pole structure of strong alternating-sign vortices. It is not clear what determines $n$; examination of other cross-sections shows that it changes as the flow evolves.

The vortices appear to retain a substantial core structure (unlike the reconnection scenarios described in other calculations $[24,25]$ which report the collapse and flattening of the vortex cores). There are also regions of tightly folded, almost crumpled, alternating sign vortex sheets, but these do not appear to be associated with the primary intensification. The results are consistent with the heuristic description proposed by Chorin and elucidated in simple lattice models of vorticity distributions [9]: a collection of vortices stretches, the local velocity fields induced by its elements intensifies, the filaments kink in order to permit the merger of countersign patches so that energy conservation can be satisfied, and the process repeats itself, cascading to smaller and smaller scales. 


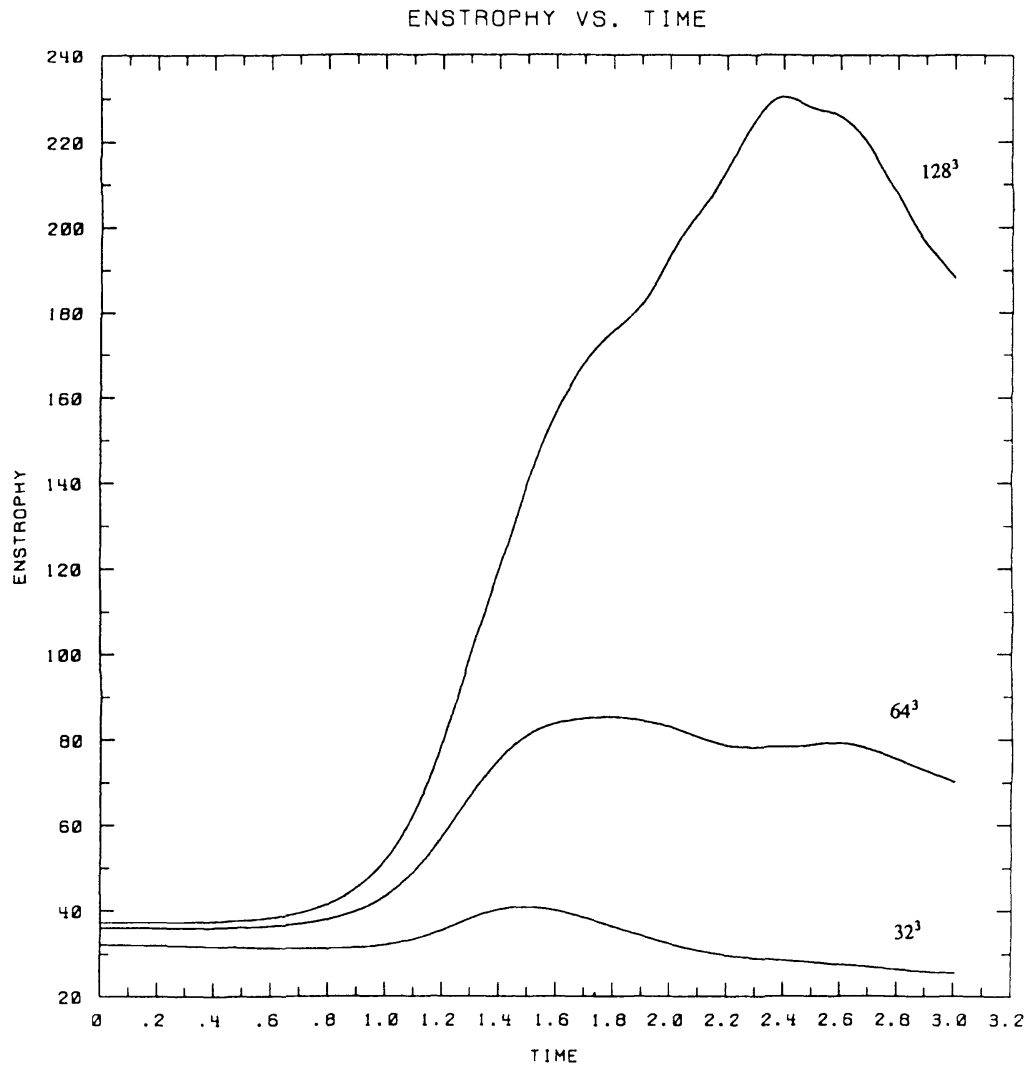

Fig. 5. Enstrophy vs. time; $32^{3}, 64^{3}$, and $128^{3}$ grids

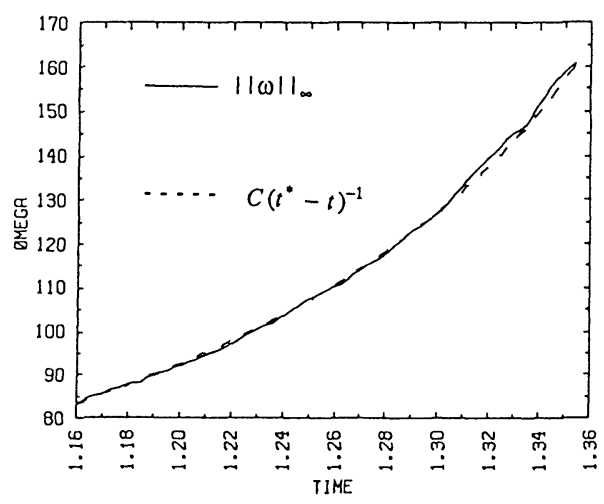

Fig. 6. Comparison of vorticity intensification with nonintegrable test function 


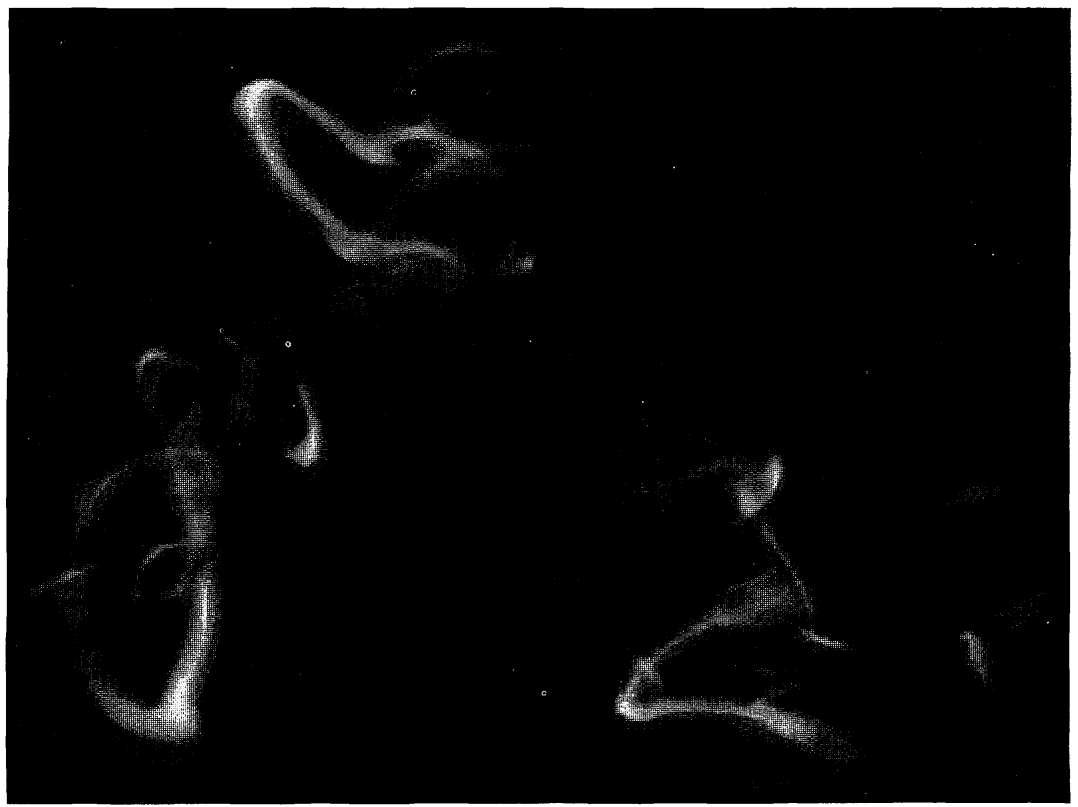

Fig. 7. Volume rendering of vorticity magnitude at $t=1.5$

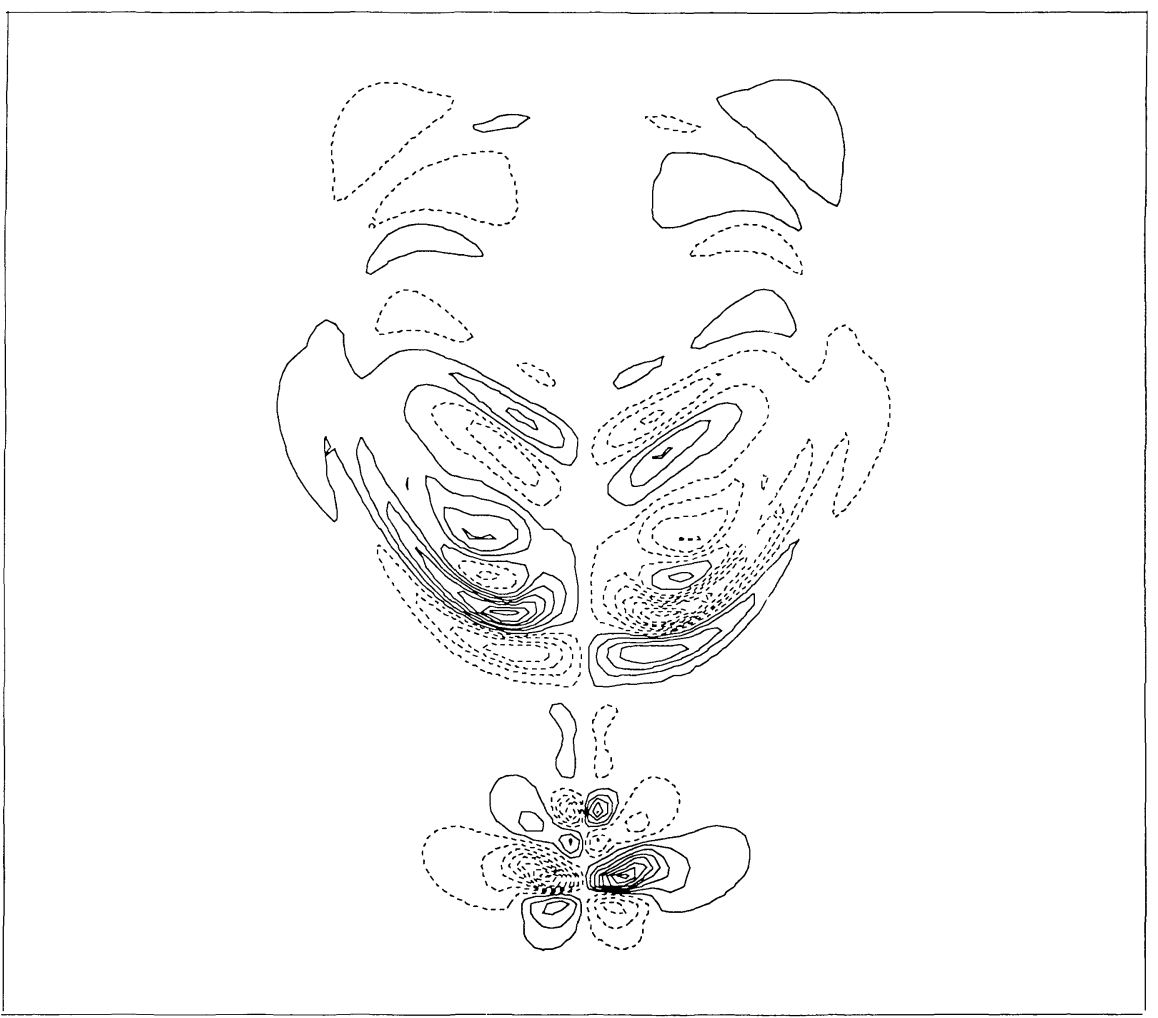

Fig. 8. $\omega_{x}\left(y, z ; X_{\omega_{\max }}\right)$ 


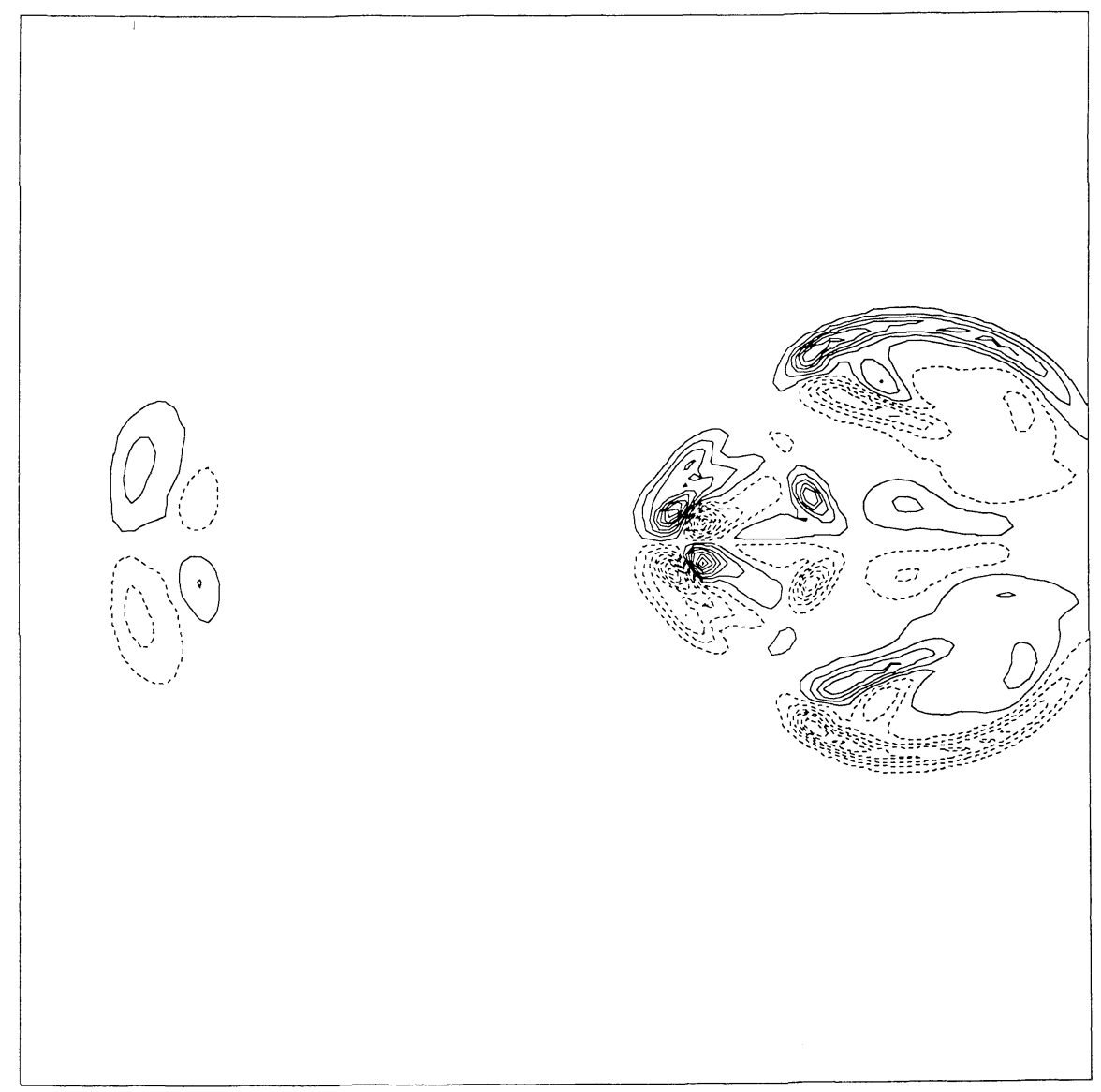

Fig. 9. $\omega_{z}\left(x, y ; Z_{\omega_{\max }}\right)$

\subsection{Energy}

The mean kinetic energy is shown in Fig. 10 for the different levels of grid resolution. It remains constant in the early stages of the growth of the perturbation, then begins to decay as the vortex tube begins to strongly selfinteract. The onset of the decay is delayed as the grid is refined; the steeper slope at late time in the high resolution $\left(128^{3}\right)$ case can be attributed to the influence of the second $L^{\infty}$ peak, which is under-resolved on the coarser grids. We presume that it is this dissipation, induced by the vorticity intensification, that permits the reconnection events we observe.

In previous calculations with the two-dimensional analog of the initial data used here, our method has been shown to conserve energy to within $0.5 \%$ and exhibits second-order convergence [4]. It is therefore unlikely that the dramatic dissipation we observe in this calculation is a numerical artifact. Indeed, the onset of decay in the kinetic energy appears to be coupled to the onset of the vorticity intensification. 
KINETIC ENERGY VS. TIME

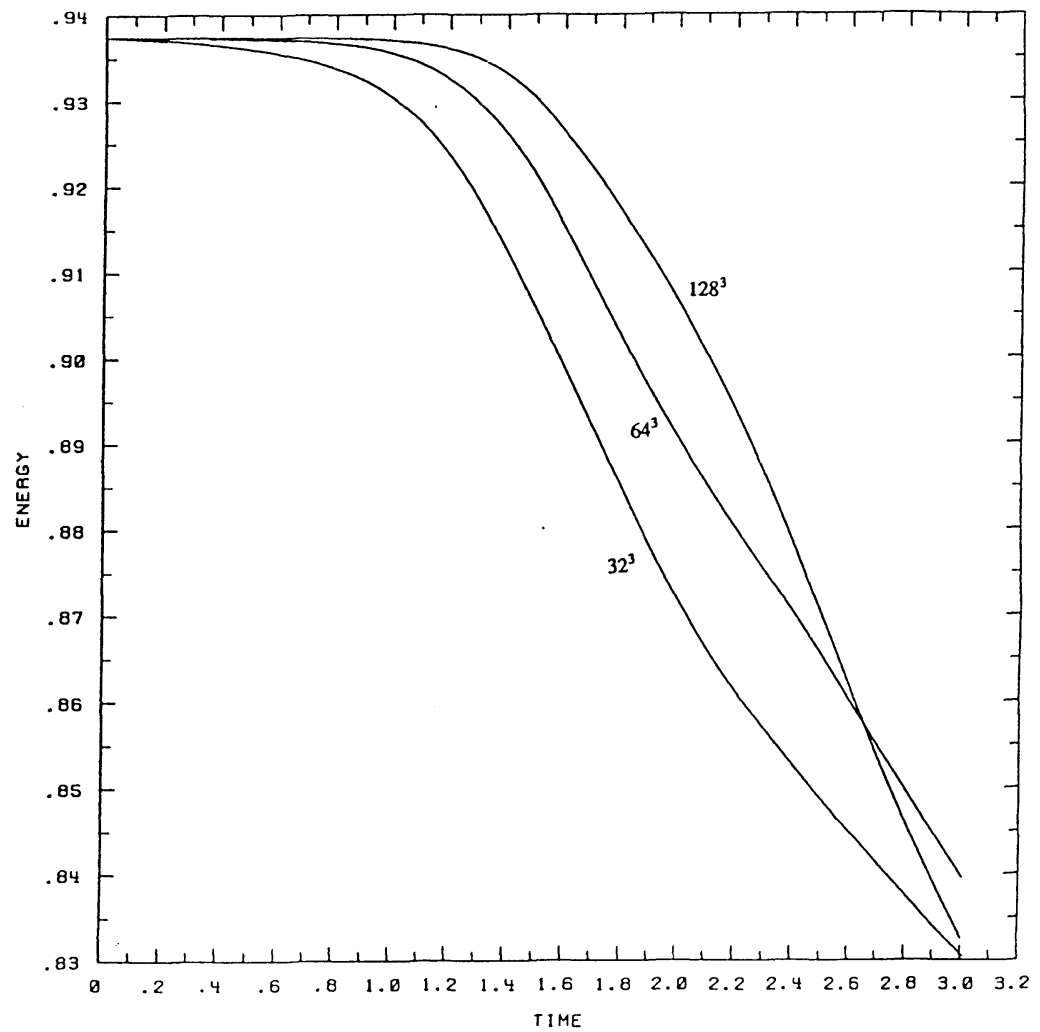

Fig. 10. Mean kinetic energy vs. time; $32^{3}, 64^{3}$, and $128^{3}$ grids

The occurrence of a singularity or near-singularity or near-singularity is indicative of a model breakdown; in the absence of such a singularity, the threedimensional Euler equations conserve energy. Viscous dissipation, therefore, serves as a physical regularization mechanism. Whether the dissipative onset we observe occurs as a manifestation of a possible breakdown of the Euler equations in the continuum limit, or if it is a signature of under-resolution alone, is not known. In any case, it highlights the intimate connection between incipient singularities and energy transfer to the small scales.

Regularization must occur; as adjacent patches of countersign vorticity merge, the energy is fluxed into increasingly high wavenumbers, which cannot be represented on a finite grid. In our calculation, the energy in these modes is then dissipated numerically. The local and selective dissipation introduced by our method at the grid scales is, in effect, a high wavenumber sink, analogous to physical dissipation at viscous length scales. (Chorin has hypothesized a different regularization scenario; namely, vortex reconnection as a manifestation of a percolation threshold for the Euler equations [13]. It is unknown if the regularization we observe can be so described, but such a mechanism is not precluded by dissipation due to the finite grid, which is clearly also present.) 


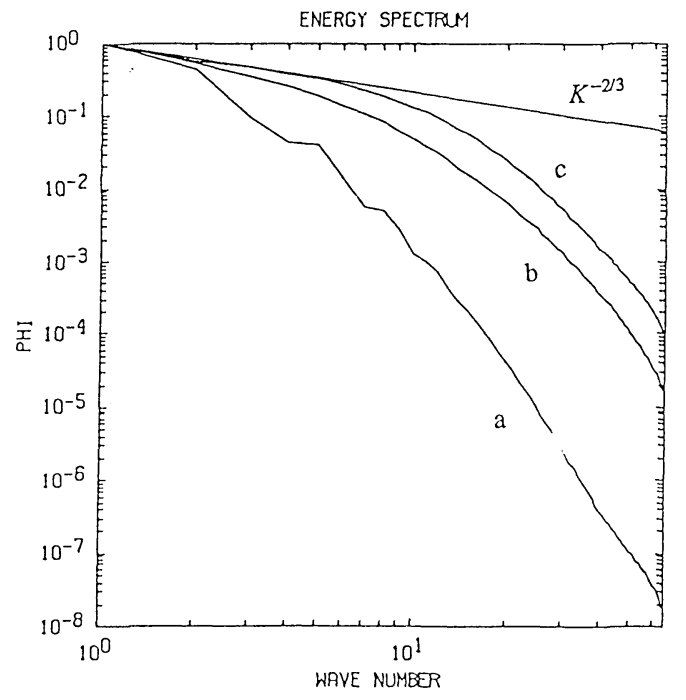

Fig. 11. Integrated energy spectrum; $a t=0.62, b t=1.65, c t=2.67$

\subsection{Energy Spectrum}

To analyze the distribution of energy as a function of wave number we define an integrated energy spectrum

$$
\Phi(K)=\int_{\vec{k}<K} E(\vec{k}) d k,
$$

where $E(\vec{k})$ is the energy at wave number vector $\vec{k}$, which is obtained by an FFT of the velocity field. Thus,

$$
\frac{d \Phi(K)}{d K}=\int_{|\vec{k}|=K} E(\vec{k}) d k
$$

is the traditional energy spectrum defined as the integral of the energy over spherical shells in $k$-space. The choice of $\Phi$ to represent the spectral data avoids numerical artifacts arising from using finite thickness shells to evaluate (2) on a discrete lattice. Figure 11 shows $\Phi(K)$ at times $0.62,1.65$, and 2.67, corresponding to before, near, and after the onset of the first singularity. We observe the growth of a region in which $\Phi(K) \approx K^{-2 / 3}$ and a steepening drop-off at high wave numbers, indicating the emergence of a Kolmogorov $\left(K^{-5 / 3}\right)$ range in the energy spectrum. At the last time shown the inertial range spans roughly a decade in wavenumber.

\subsection{Moments of the Strain}

In order to examine the statistical properties of the spatial distribution of strain rate in the flow, and to elucidate its relationship to the vorticity distribution, we calculate the moments of the diagonal components of the deformation tensor

$$
e_{i j}=\frac{1}{2}\left(\frac{\partial u_{i}}{\partial x_{j}}+\frac{\partial u_{j}}{\partial x_{i}}\right) \text {. }
$$




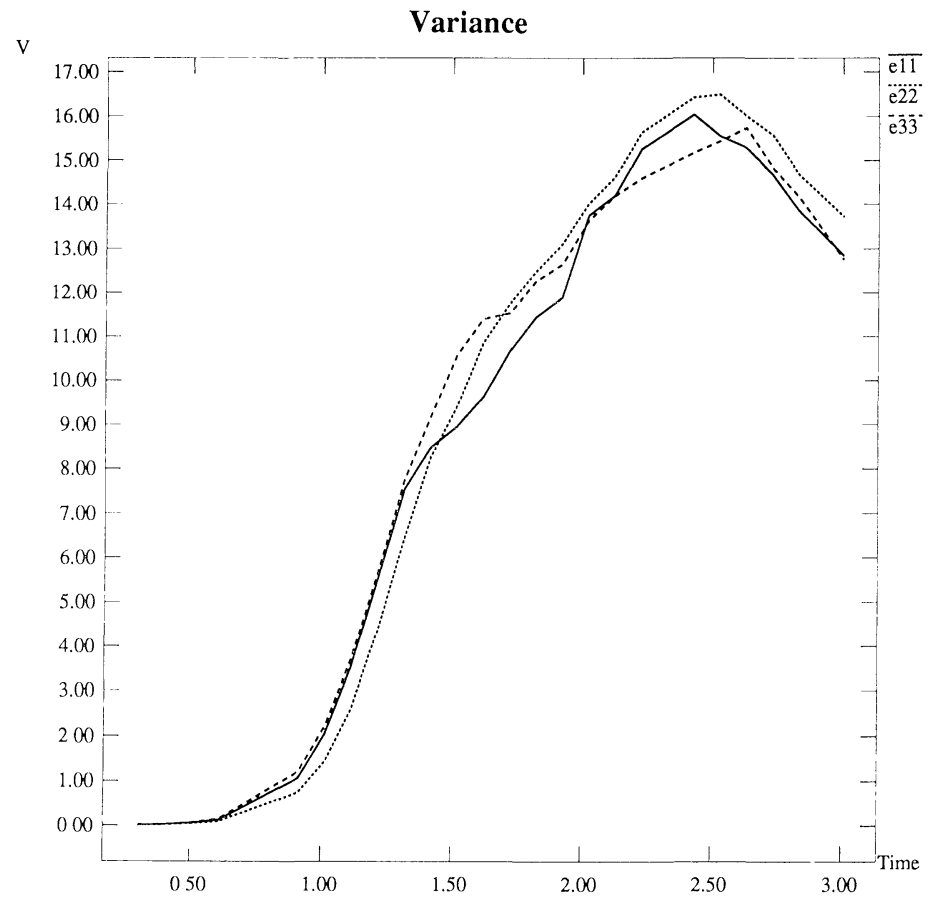

Fig. 12. Variance of $e_{i i}$ vs. time

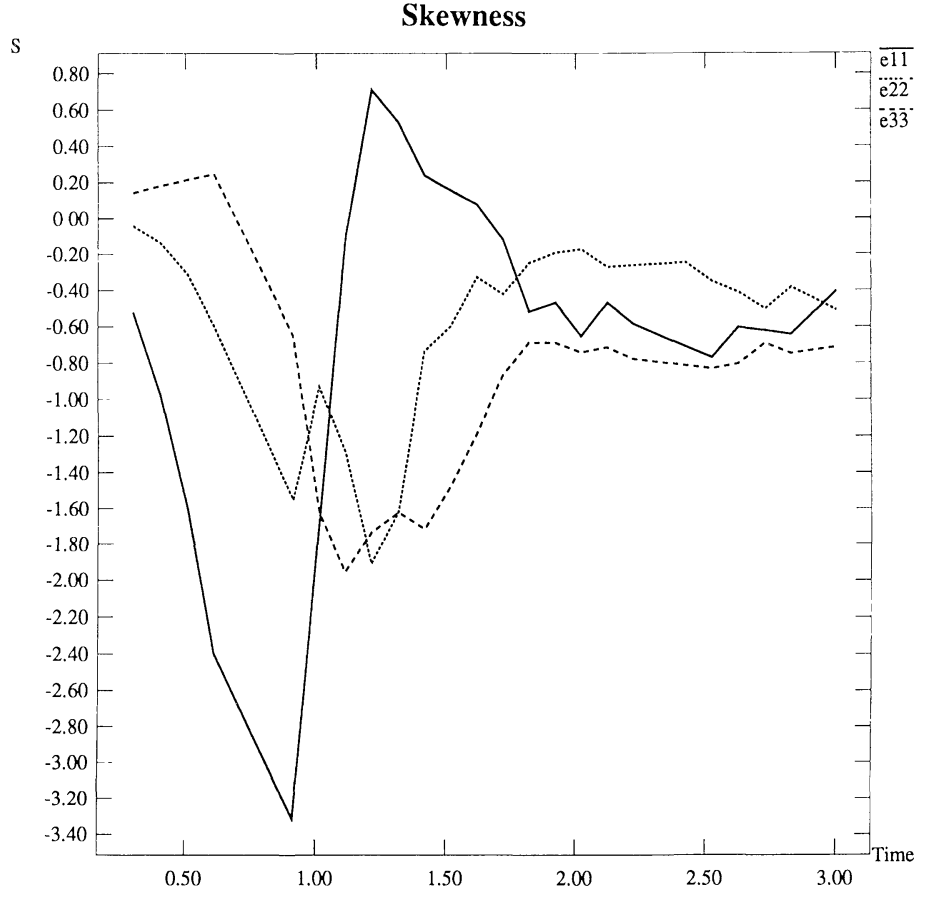

Fig. 13. Skewness of $e_{i t}$ vs. time 


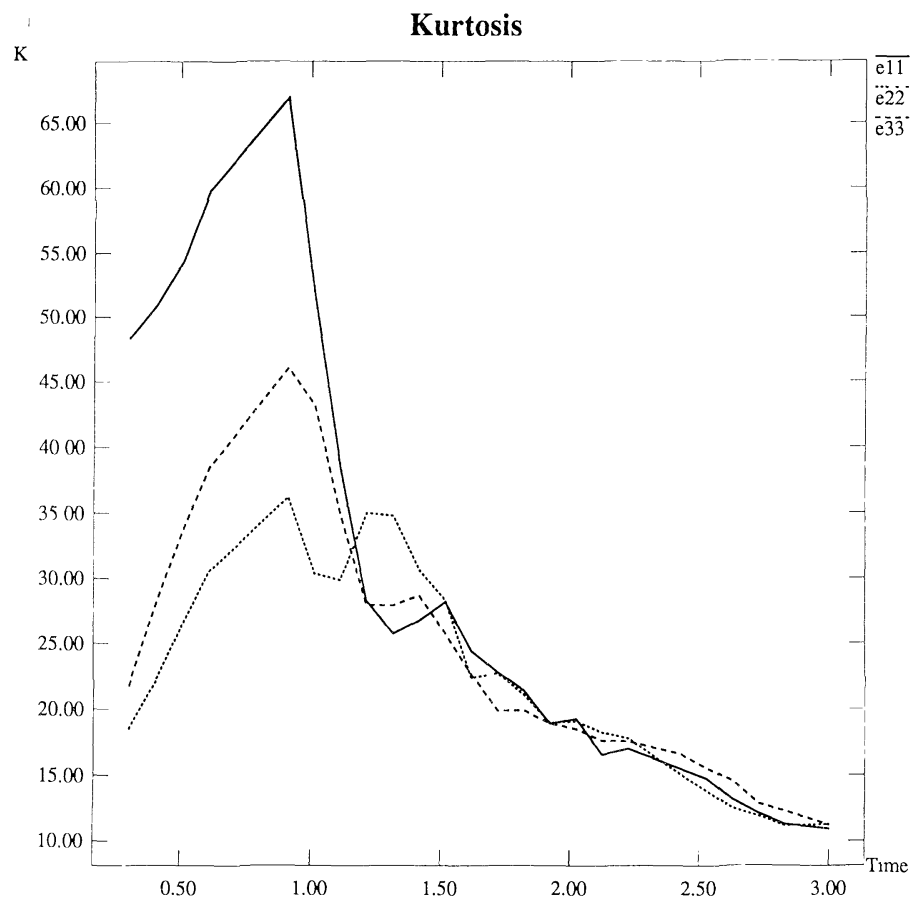

Fig. 14. Kurtosis of $e_{l l}$ vs. time

Figure 12 shows the time evolution of the variance, given for a random variable $\chi$ with zero mean by $V(\chi)=\left\langle\chi^{2}\right\rangle$. It is a measure of the width of the probability density function $P(\chi)$. The components appear to roughly obey an equipartition principle. The increase in variance can be interpreted as a signature of the generation of increasingly energetic small scales. The curves show a striking resemblance to the enstrophy.

The skewness, $S(\chi)$, is given by $S(\chi)=\left\langle\chi^{3}\right\rangle /\left\langle\chi^{2}\right\rangle^{3 / 2}$ and is a measure of the symmetry of $P(\chi)$. If $\chi$ is Gaussian, $S(\chi)=0$. In a turbulent flow, skewness indicates the rate at which enstrophy is increased by vortex stretching. Batchelor and Townsend [2] have reported experimental values of -0.4 ; numerical simulations at moderate Reynolds number have yielded values of -0.5 [21]. In Fig. 13 we show the time evolution of the skewness of $e_{i i}$. The $u$-component in particular shows wild variation in the early stages of the flow; eventually all three components relax to values between -0.2 and -0.6 . The statistics are clearly nonGaussian.

In Fig. 14 we show the kurtosis, or flatness, of $e_{i i}$, defined by $K(\chi)=\left\langle\chi^{4}\right\rangle /\left\langle\chi^{2}\right\rangle^{2}$. The magnitude of the kurtosis is an indication of the peakiness of $\chi$, or magnitude of the values that $P(\chi)$ takes on in its tails. Again, the $u$-component shows a violent initial increase. At late times, all three components relax to a value well in excess of the Gaussian value of 3. The rapid and extreme variations in skewness and kurtosis could be related to the turbulent bursts observed in boundary layers and shear flows. 


\subsection{Entropy}

If we partition the magnitude of the vorticity on the grid $\left|\omega_{i j k}\right|$ into $L$ equal bins of size $\delta_{\omega}$, we can define a probability density function by

$$
P_{l}=\sum_{i j k} \mu\left(\omega_{i j k}, l\right)
$$

where

$$
\mu\left(\omega_{i j k}, l\right)= \begin{cases}N^{-3} & \text { if }\left|\omega_{l}-\omega_{i j k}\right| \leqq \delta_{\omega} / 2, \\ 0 & \text { otherwise }\end{cases}
$$

with $\omega_{l}=l \delta_{\omega}$ and $l=1, \ldots, L$.

An entropy of the vorticity distribution is then given by

$$
S=-\sum_{l} P_{l} \ln P_{l} .
$$

If an appeal is made to statistical descriptions of polymers, this quantity can be regarded as a measure of line length $[16,12]$. Therefore, the entropy of a collection of vortices measures the severity of vortex stretching, and is closely related to the enstrophy. The more conventional interpretation of entropy as the number of degrees of freedom needed to specify the state of the system is also appropriate here, as evidenced by the emergence of an inertial range.

Figure 15 shows the time evolution of $S$ for this flow. The entropy increases gradually at first, then sharply, beginning to level off where the enstrophy reaches

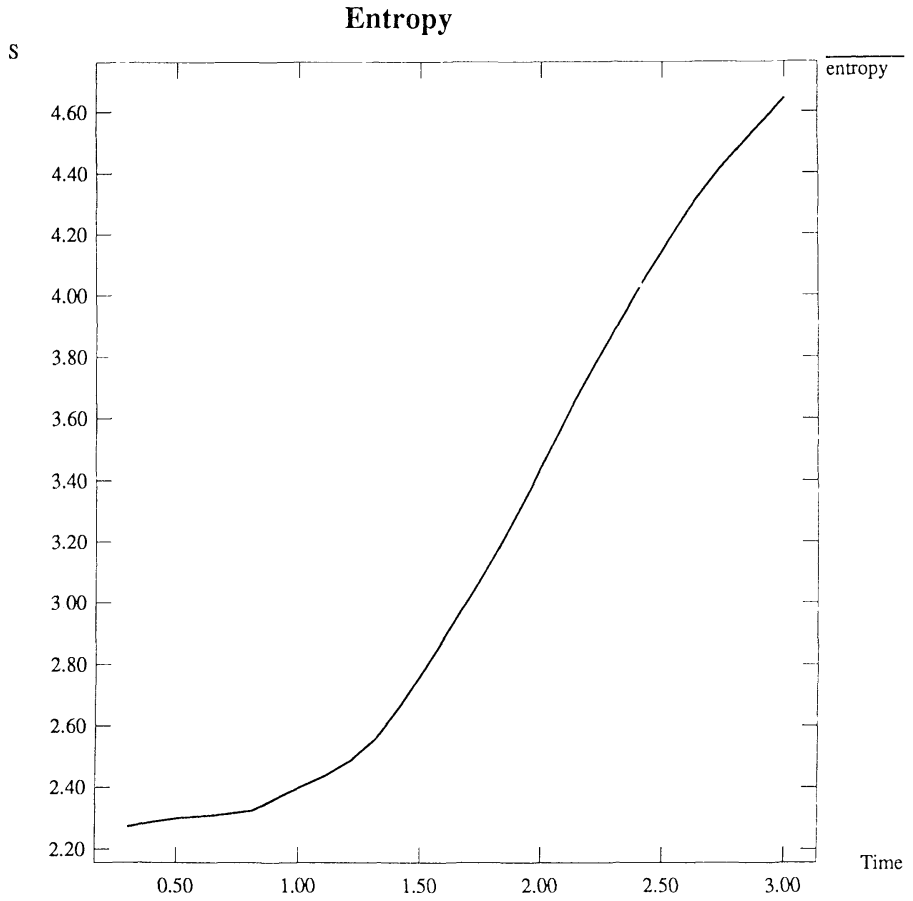

Fig. 15. Entropy vs. time 
its maximum value. We have not carried out the calculation far enough to determine if the entropy tends to some asymptotic value. This is not expected, however. The maximum entropy state would correspond to a quasi-equilibrium inertial range, in which energy is dissipated at the small scales at the same rate it is injected at the large scales [13]. Here we are introducing no energy at low $k$ and at late times the high wavenumber modes will slowly decay due to grid dissipation.

\section{Conclusion}

We have carried out a numerical solution of the three-dimensional Euler equations for the evolution of a perturbed vortex tube, and shown that strong vorticity intensification events play a fundamental role in the dynamics. We have discovered a new mechanism for such events; namely, that the intensification is concentrated on tightly curved and crumpled regions of alternating sign vorticity. This constitutes an instantiation of Chorin's lattice models of vorticity distributions. The intensification in $L^{\infty}$ becomes, to the limit of grid resolution, unbounded as $t$ approaches a critical time $t^{*}$ and is nonintegrable. The onset of the intensification is accompanied by a decay in the kinetic energy, suggesting that the intensification is mitigated by viscous regularization in a manner analogous to shocks in Burgers' equation. The velocity statistics are highly non-Gaussian. The enstrophy and entropy continue to increase beyond the first $L^{\infty}$ peak, elucidating the continuing importance of vortex stretching. At late times we witness the emergence of roughly a decade of Kolmogorov range.

Suggestive as this calculation is, the question of a finite-time singularity in the three-dimensional Euler equations remains open. What we have done is shed light on the structure and role of vorticity intensification events in transition to turbulence. Ongoing work in this area includes an enhanced resolution study of the $2 n$-pole structure in the intensification region, careful analysis of the local coupling of vorticity and strain, and implementation of the projection algorithm in an adaptive mesh refinement shell to increase the effective resolution and enhance computational efficiency.

Acknowledgements. This work was performed under the auspices of the U.S. Department of Energy by the Lawrence Livermore National Laboratory under contract No. W-7405-Eng-48. Partial support under contract No. W-7405-Eng-48 was provided by the Applied Mathematical Sciences Program of the Office of Energy Research. DLM would like to thank the Institute for Advanced Study, Princeton, NJ, for their hospitality during the completion of this paper.

\section{References}

1. Ashurst, W., Meiron, D.: Numerical study of vortex reconnection. Phys. Rev. Lett. 58, 1632 (1987)

2. Batchelor, G.K., Townsend, A.A.: Decay of vorticity in isotropic turbulence. Proc. R. Soc. A 191, 534-550 (1947)

3. Beale, J.T., Kato, T., Majda, A.: Remarks on the breakdown of smooth solutions for the 3-D Euler equations. Commun. Math. Phys. 94, 61-66 (1989)

4. Bell, J.B., Colella, P., Glaz, H.: A second-order projection method for the incompressible Navier-Stokes equations. J. Comp. Phys. 257-283 (1989)

5. Bell, J.B., Solomon, J.M., Szymczak, W.G.: A second-order projection method for the threedimensional Euler and Navier-Stokes equations. Preprint 1990 
6. Brachet, M., Meiron, D., Orszag, S., Nickel, B., Morf, R., Frisch, U.: Small-scale structure of the Taylor-Green vortex. J. Fluid Mech. 130, 411 (1983)

7. Chorin, A.J.: Numerical solution of the Navier-Stokes equations. Math. Comp. 22, 745-762 (1968)

8. Chorin, A.J.: The evolution of a turbulent vortex. Commun. Math. Phys. 83, 517-535 (1982)

9. Chorin, A.J.: Turbulence and vortex stretching on a lattice. Commun. Pure Appl. Math. 39, S47-S65 (1986)

10. Chorin, A.J.: Hairpin removal in vortex interaction. LBL-26173, Lawrence Berkeley Laboratory Report, 1988

11. Chorin, A.J., Akao, J.: Vortex equilibria in turbulence and quantum analogues. Physica D, 1991 (in press)

12. Chorin, A.J.: Statistical mechanics and vortex motion. PAM-500, Center for Pure and Applied Mathematics Report, University of California, Berkeley 1990

13. Chorin, A.J.: Equilibrium statistics of a vortex filament with applications. LBL-30419, Lawrence Berkeley Laboratory Report, 1991

14. Colella, P.: Multidimensional upwind methods for hyperbolic conservation laws. J. Comp. Phys. 94, 61-66 (1990)

15. Falco, R.E.: Phys. Fluids 20, S124 (1977)

16. de Gennes, P.G.: Scaling Concepts in Polymer Physics, Ithaca, NY: Cornell University Press 1971

17. Kerr, R.M., Hussain, F.: Simulation of vortex reconnection. Physica D 37, 474-484 (1989)

18. Kim, J., Moin, P.: The structure of the vorticity field in turbulent channel flow. Part 2. Study of ensemble averaged fields. J. Fluid Mech. 162, 339 (1986)

19. Moin, P., Leonard, A., Kim, J.: Evolution of a curved vortex filament into a vortex ring. Phys. Fluids 29, 955-963 (1986)

20. Moin, P., Rogers, M.M., Moser, R.D.: Proceedings of the Fifth Symposium on Turbulent Shear Flows, Cornell University, Ithaca, NY, 1985

21. Numerical simulations of turbulence. In: Rosenblatt, M., Van Atta, C. (eds.). Statistical Models of Turbulence, pp. 127-147. Berlin, Heidelberg, New York: Springer 1972

22. Pumir, A., Kerr, R.M.: Numerical simulations of interacting vortex tubes. Phys. Rev. Lett. 58, $1636(1987)$

23. Pumir, A., Siggia, E.D.: Vortex dynamics and the existence of solutions to the Navier-Stokes equations. Phys. Fluids 30, 1606-1626 (1987)

24. Pumir, A., Siggia, E.D.: Collapsing solutions to the 3-D Euler equations. Phys. Fluids A 2 (3), 220-241 (1990)

25. Shelley, M.J., Meiron, D.I.: Vortex reconnection and smoothness of the Euler equations. In: Anderson, C., Greengard, C. (eds.). Lectures in Applied Mathematics, AMS, 1991

26. Siggia, E.D.: Collapse and amplification of a vortex filament. Phys. Fluids 28, 794-805 (1985)

27. Zabusky, N.J., Melander, M.V.: Three-dimensional vortex tube reconnection: Morphology for orthogonally offset tubes. Physica D 37, 555-562 (1989)

Communicated by S.-T. Yau 